\title{
Mesons and glueballs in chiral approach and AdS/QCD
}

\author{
Valery E. Lyubovitskij $;$ Tanja Branz, Amand Faessler, Thomas Gutsche \\ Institut für Theoretische Physik, Universität Tübingen, \\ Kepler Center for Astro and Particle Physics, \\ Auf der Morgenstelle 14, D-72076 Tübingen, Germany \\ E-mail: valeri.lyubovitskij@uni-tuebingen.de \\ E-mail: tanja.branz@uni-tuebingen.de \\ E-mail: amand.faesslereuni-tuebingen.de \\ E-mail: thomas.gutsche@uni-tuebingen.de
}

\begin{abstract}
Phenomenology of mesons and glueballs is considered in two frameworks - chiral approach lightfront holographic approach proposed by Brodsky and Teramond, which is based on the correspondence of string theory in Anti-de Sitter and conformal field theory in physical space-time.
\end{abstract}

6th International Workshop on Chiral Dynamics, CD09

July 6-10, 2009

Bern, Switzerland

\footnotetext{
* Speaker.

†n leave of absence from Department of Physics, Tomsk State University, 634050 Tomsk, Russia
} 


\section{Introduction}

Phenomenology of mesons and glueballs is considered in two frameworks - chiral approach [1, 2] and light-front holographic (LFH) approach proposed by Brodsky and Teramond [3], which is based on the correspondence of string theory in Anti-de Sitter (AdS) and conformal field theory in physical space-time.

First we report our results on strong and electromagnetic decay properties of scalar, pseudoscalar, vector and tensor mesons above $1 \mathrm{GeV}$ within a chiral approach [1]. 包. The isoscalar states are treated as mixed states of quarkonia and glueball configurations. A fit to the experimental mass and decay rates listed by the Particle Data Group is performed to extract phenomenological constraints on the nature of the meson resonances and to the issue of the glueballs decays. A comparison to other experimental and theoretical results and possible hints for exotic mesons and open interpretation-issues are discussed.

Second we present summary of our results for mass spectroscopy and decay constants of light and heavy mesons [ 4 , 5] in the LFH approach [3]. The LFH approach is a covariant and analytic model for hadron structure with confinement at large distances and conformal behavior at short distances. It is analogous to the Schrödinger theory for atomic physics and provides the precise mapping of string modes $\Phi(z)$ in the AdS fifth dimension $z$ to the hadron light-front wave functions in physical space-time in terms of light-front impact variable $\zeta$, which measures the separation of the quark and gluonic constituents inside a hadron.

\section{Mesons and glueballs in chiral approach}

In this work, starting from an effective chiral Lagrangian [1, 2] derived in Chiral Perturbation Theory (ChPT) [6], we perform a tree-level analysis of the strong and electromagnetic decays of scalar, pseudoscalar and vector mesons settled in the energy range between 1 and $2 \mathrm{GeV}$. Although chiral approach cannot be rigorously justified at this energy scale, since loop corrections could be large, we intend to use this framework as a phenomenological tool to extract possible glueball-quarkonia mixing scenarios from the observed decays. The scalar glueball is introduced as an extra-flavor singlet composite field with independent couplings to pseudoscalar mesons (and to photons, although suppressed). In particular, we follow the assignment that the bare quarkonia states $N=(\bar{u} u+\bar{d} d) / \sqrt{2}, S=\bar{s} s$ and the bare scalar glueball $G$ mix, resulting in the three scalar-isoscalar resonances $f_{0}(1370), f_{0}(1500)$ and $f_{0}(1710)$. Such a mixing scheme has been previously investigated by many authors (see e.g. discussion in [2]). The $\eta(1405)$ meson is considered as a strong candidate for the pseudoscalar glueball. Also we take into account it mixture with $\eta(1295) \approx n \bar{n}$ and $\eta(1475) \approx s \bar{s}$ states. Here we present results for the scalar mesons/glueball (Table I) and tensor (Table II) mesons. Further details can be found in Refs. [1], 2].

\section{Light and heavy mesons in holographic approach}

In series of papers (see e.g. [3]) Brodsky and Teramond developed a semiclassical approximation to QCD - light-front holography (LFH) approach based on correspondence of string theory in Anti-de Sitter (AdS) space and conformal field theory (CFT) in physical space-time [7]. Light-front 
holography [3] is one of the exciting features of AdS/CFT correspondence. The LFH approach is a covariant and analytic model for hadron structure with confinement at large distances and conformal behavior at short distances. It is analogous to the Schrödinger theory for atomic physics. It provides the precise mapping of string modes $\Phi(z)$ in the AdS fifth dimension $z$ to the hadron light-front wave functions (LFWF) in physical space-time in terms of light-front impact variable $\zeta$ which measures the separation of the quark and gluonic constituents inside a hadron. Therefore, different values of the holographic variable $z$ correspond to different scales at which the hadron is examined. The mapping was obtained by matching the matrix elements (e.g. electromagnetic pion form factor, the energy-momentum tensor) in the two approaches - string theory in AdS and light-front theory in Minkowski space-time. In this section we consider application of holographic approach for decay properties of light and heavy mesons.

Table I. Masses and decay properties of scalar mesons and scalar glueball

\begin{tabular}{|l|l|l|l|}
\hline Quantity & Exp & Theory & $\chi_{i}^{2}$ \\
\hline$M_{f_{1}}(\mathrm{MeV})$ & $1350 \pm 150$ & 1417 & 0.202 \\
\hline$M_{f_{2}}(\mathrm{MeV})$ & $1507 \pm 5$ & 1507 & $\sim 0$ \\
\hline$M_{f_{3}}(\mathrm{MeV})$ & $1714 \pm 5$ & 1714 & 0.003 \\
\hline$\Gamma_{f_{2} \rightarrow \pi \pi}(\mathrm{MeV})$ & $38.0 \pm 4.6$ & 38.52 & 0.011 \\
\hline$\Gamma_{f_{2} \rightarrow \bar{K} K}(\mathrm{MeV})$ & $9.4 \pm 1.7$ & 10.36 & 0.322 \\
\hline$\Gamma_{f_{2} \rightarrow \eta \eta}(\mathrm{MeV})$ & $5.6 \pm 1.3$ & 1.90 & 8.109 \\
\hline$\Gamma_{f_{3} \rightarrow \pi \pi} / \Gamma_{f_{3} \rightarrow \bar{K} K}$ & $0.20 \pm 0.06$ & 0.212 & 0.036 \\
\hline$\Gamma_{f_{3} \rightarrow \eta \eta} / \Gamma_{f_{3} \rightarrow \bar{K} K}$ & $0.48 \pm 0.15$ & 0.249 & 2.446 \\
\hline$\Gamma_{a_{0} \rightarrow \bar{K} K} / \Gamma_{a_{0} \rightarrow \pi \eta}$ & $0.88 \pm 0.23$ & 0.838 & 0.032 \\
\hline$\Gamma_{a_{0} \rightarrow \pi \eta^{\prime}} / \Gamma_{a_{0} \rightarrow \pi \eta}$ & $0.35 \pm 0.16$ & 0.288 & 0.150 \\
\hline$\Gamma_{K_{0}^{*} \rightarrow K \pi}(\mathrm{MeV})$ & $273 \pm 51$ & 59.10 & 17.590 \\
\hline$\left(\Gamma_{f_{3}}\right)_{2 P}(\mathrm{MeV})$ & $140 \pm 10$ & 143.27 & 0.110 \\
\hline$\chi_{\text {tot }}^{2}$ & - & - & 29.01 \\
\hline
\end{tabular}

Table II. Decay properties of tensor mesons

\begin{tabular}{|l|l|l|l|}
\hline Mode & Exp (MeV) & Theory $(\mathrm{MeV})$ & $\chi_{i}^{2}$ \\
\hline$\Gamma_{f_{2} \rightarrow \pi \pi}$ & $157.0 \pm 7.6$ & 153.51 & 0.210 \\
\hline$\Gamma_{f_{2} \rightarrow \bar{K} K}$ & $8.5 \pm 0.9$ & 9.15 & 0.526 \\
\hline$\Gamma_{f_{2} \rightarrow \eta \eta}$ & $0.83 \pm 0.20$ & 0.80 & 0.023 \\
\hline$\Gamma_{f_{2}^{\prime} \rightarrow \pi \pi}$ & $0.60 \pm 0.16$ & 0.55 & 0.102 \\
\hline$\Gamma_{f_{2}^{\prime} \rightarrow \bar{K} K}$ & $64.8 \pm 7.6$ & 41.64 & 9.288 \\
$\Gamma_{f_{2}^{\prime} \rightarrow \eta \eta}$ & $7.5 \pm 2.9$ & 6.49 & 0.196 \\
\hline$\Gamma_{a_{2} \rightarrow \bar{K} K}$ & $5.2 \pm 1.1$ & 6.64 & 1.716 \\
\hline$\Gamma_{a_{2} \rightarrow \eta \pi}$ & $15.5 \pm 2.0$ & 18.42 & 2.134 \\
\hline$\Gamma_{a_{2} \rightarrow \eta^{\prime} \pi}$ & $0.57 \pm 0.12$ & 0.80 & 3.652 \\
\hline$\Gamma_{K *_{2} \rightarrow \bar{K} K}$ & $49.1 \pm 2.5$ & 40.08 & $\sim 0$ \\
\hline$\chi_{t o t}^{2}$ & - & - & 18.496 \\
\hline
\end{tabular}


Our starting point is the equation of motion (EOM) for the AdS mode in the fifth dimension $z$ (holographic coordinate). The soft-wall model developed in Ref. [3] is based on the following one-dimensional Schrödinger EOM:

$$
\left[-\frac{d^{2}}{d z^{2}}-\frac{1-4 L^{2}}{4 z^{2}}+U(z)\right] \Phi(z)=M^{2} \Phi_{1}(z)
$$

where $U(z)$ is the effective confinement potential:

$$
U(z)=\kappa^{4} z^{2}+2 \kappa^{2}(L+S-1) .
$$

$n, L, S$ are the radial, orbital and spin quantum numbers, respectively; $\kappa$ is the scale parameter related to the dilaton field.

The string mode is given by normalizable solution

$$
\Phi(z)=\sqrt{\frac{2 n !}{(n+L) !}} \kappa^{1+L} z^{\frac{1}{2}+L} e^{-\frac{1}{2} \kappa^{2} z^{2}} L_{n}^{L}\left(\kappa^{2} z^{2}\right)
$$

with $\int_{0}^{\infty} d z \Phi^{2}(z)=1$. Here $L_{n}^{L}(x)$ is the generalized Laguerre polynomials. From Eqs. 3.1) we get the hadron spectrum at zero quark masses

$$
M^{2}=4 \kappa^{2}\left(n+L+\frac{S}{2}\right)
$$

$\Phi(z)$ can be directly mapped to the LFWF due to correspondence of AdS and light-front amplitudes. In particular, considering the case with two partons $q_{1}$ and $\bar{q}_{2}$ and making correspondence of the holographic coordinate $z$ to the impact variable $\zeta$ in the LF formalism

$$
z \rightarrow \zeta, \quad \zeta^{2}=\mathbf{b}_{\perp}^{2} x(1-x)
$$

where $\mathbf{b}_{\perp}$ is the impact separation and Fourier conjugate to the transverse momentum $\mathbf{k}_{\perp}$, we obtain the relation between the AdS mode and meson LFWF $\widetilde{\psi}_{q_{1} \bar{q}_{2}}(x, \zeta)$ in massless case:

$$
\left|\widetilde{\psi}_{q_{1} \bar{q}_{2}}(x, \zeta)\right|^{2}=x(1-x) f^{2}(x) \frac{|\Phi(\zeta)|^{2}}{2 \pi \zeta}
$$

where $f(x)=1$. Extension to massive quarks has been suggested by Brodsky and Teramond [3] and later was considered in [5]:

$$
\begin{aligned}
& -\frac{d^{2}}{d \zeta^{2}} \rightarrow-\frac{d^{2}}{d \zeta^{2}}+\mu_{12}^{2}, \quad \mu_{12}^{2}=\frac{m_{1}^{2}}{x}+\frac{m_{2}^{2}}{1-x} \\
& f(x) \rightarrow f\left(x, m_{1}, m_{2}\right) \equiv N f(x) e^{-\frac{\mu_{12}^{2}}{2 \lambda^{2}}},
\end{aligned}
$$

where $N$ is normalization constant, $\lambda$ is additional scale parameter.

The modified hadronic wave function and mass spectrum are written as:

$$
\begin{aligned}
& \psi_{q_{1} \bar{q}_{2}}\left(x, \zeta, m_{1}, m_{2}\right)=\frac{\Phi(\zeta)}{\sqrt{2 \pi \zeta}} f\left(x, m_{1}, m_{2}\right) \sqrt{x(1-x)} \\
& M^{2}=\int_{0}^{\infty} d \zeta \Phi(\zeta)\left(-\frac{d^{2}}{d \zeta^{2}}-\frac{1-4 L^{2}}{4 \zeta^{2}}+U(\zeta)\right) \Phi(\zeta)+\int_{0}^{1} d x\left(\frac{m_{1}^{2}}{x}+\frac{m_{2}^{2}}{1-x}\right) f^{2}\left(x, m_{1}, m_{2}\right)
\end{aligned}
$$


One should stress that this approach correctly reproduces mass spectrum of heavy-light mesons in the heavy quark limit

$$
\begin{aligned}
M_{Q q}^{2} & =4 \kappa^{2}\left(n+L+\frac{S}{2}\right)+\int_{0}^{1} d x\left(\frac{m_{q}^{2}}{x}+\frac{m_{Q}^{2}}{1-x}\right) f^{2}\left(x, m_{q}, m_{Q}\right)+\cdots \\
& =\left(m_{Q}+\bar{\Lambda}+\mathscr{O}\left(1 / m_{Q}\right)\right)^{2}
\end{aligned}
$$

where dimensional parameters are scaled as $\kappa=\mathscr{O}\left(m_{Q}^{0}\right)$ and $\lambda=\mathscr{O}\left(\sqrt{m_{Q}}\right)$. It is seen that Eq. (3.9) is in agreement with prediction of heavy quark effective theory (HQET):

$$
M_{V}-M_{P}=\frac{2 \kappa^{2}}{M_{V}+M_{P}} \sim \frac{1}{m_{Q}}
$$

It is also interesting to consider the limit of heavy quark masses for heavy quarkonia $\left(Q_{1} \bar{Q}_{2}\right)$. For this we express the longitudinal momentum fractions through the $z$-component of the internal momentum $\mathbf{k}=\left(\mathbf{k}_{\perp}, k_{z}\right)$ as (see also [8]):

$$
x=\frac{e_{1}+k_{z}}{e_{1}+e_{2}}, \quad 1-x=\frac{e_{2}-k_{z}}{e_{1}+e_{2}}
$$

where $e_{i}=\sqrt{m_{Q_{i}}^{2}+\mathbf{k}^{2}}$ and $\mathbf{k}^{2}=\mathbf{k}_{\perp}^{2}+k_{z}^{2}$. Then considering heavy quark limit $m_{Q_{1}}, m_{Q_{2}} \gg \mathbf{k}_{\perp}, k_{z}$ we get

$$
x=\frac{m_{Q_{1}}+k_{z}}{m_{Q_{1}}+m_{Q_{2}}}+\mathscr{O}\left(1 / m_{Q}^{2}\right), \quad 1-x=\frac{m_{Q_{2}}-k_{z}}{m_{Q_{1}}+m_{Q_{2}}}+\mathscr{O}\left(1 / m_{Q}^{2}\right)
$$

Hence,

$$
\frac{m_{Q_{1}}^{2}}{x}+\frac{m_{Q_{2}}^{2}}{1-x}=\left(m_{Q_{1}}+m_{Q_{2}}\right)^{2}+\mathscr{O}(1)
$$

Therefore the leading term of the integral containing the longitudinal mode is simply given by $\left(m_{Q_{1}}+m_{Q_{2}}\right)^{2}$, which is the leading contribution to the mass squared of the heavy quarkonia. It means that we correctly reproduce an expansion of heavy quarkonia mass in heavy quark limit:

$$
M_{Q_{1} \bar{Q}_{2}}=m_{Q_{1}}+m_{Q_{2}}+E+\mathscr{O}\left(1 / m_{Q_{1,2}}\right),
$$

where $E$ is binding energy.

As application we present results for decay constants of pseudoscalar and vector mesons including light and heavy quarks (see Tables III-V).

Table III. Decay constants $f_{P}$ of pseudoscalar mesons

\begin{tabular}{|l|c|c|c|l|}
\hline Meson & Data $[\mathrm{MeV}]$ & $f_{P}[\mathrm{MeV}]$ & $R=f_{P} / f_{P}^{\mathrm{exp}}$ & $\kappa[\mathrm{GeV}]$ \\
\hline$\pi^{-}$ & $130.4 \pm 0.04 \pm 0.2$ & 130.4 & 1 & 0.425 \\
\hline$K^{-}$ & $155.5 \pm 0.2 \pm 0.8$ & 155.5 & 1 & 0.507 \\
\hline$D^{+}$ & $205.8 \pm 8.9$ & 182.1 & 0.88 & 0.6 \\
\hline$D_{s}^{+}$ & $273 \pm 10$ & 183 & 0.67 & 0.6 \\
\hline$B^{-}$ & $216 \pm 22$ & 165.7 & 0.77 & 0.6 \\
\hline$B_{s}^{0}$ & $253 \pm 8 \pm 7$ & 166.2 & 0.66 & 0.6 \\
\hline$B_{c}$ & $489 \pm 5 \pm 3$ & 399 & 0.82 & 1.33 \\
\hline
\end{tabular}


Table IV. Decay constants $f_{V}$ of vector mesons with open flavor

\begin{tabular}{|l|c|c|c|l|}
\hline Meson & Data $[\mathrm{MeV}]$ & $f_{V}[\mathrm{MeV}]$ & $R=f_{V} / f_{V}^{\text {exp }}$ & $\kappa[\mathrm{GeV}]$ \\
\hline$D^{*}$ & $245 \pm 20_{-2}^{+3}$ & 182.1 & 0.74 & 0.6 \\
\hline$D_{s}^{*}$ & $272 \pm 16_{-20}^{+3}$ & 183 & 0.67 & 0.6 \\
\hline$B^{*}$ & $196 \pm 24_{-2}^{+39}$ & 165.7 & 0.85 & 0.6 \\
\hline$B_{s}^{*}$ & $229 \pm 20_{-16}^{+41}$ & 166.2 & 0.73 & 0.6 \\
\hline
\end{tabular}

Table V. Decay constants $f_{V}$ of vector mesons with hidden flavor

\begin{tabular}{|l|c|c|c|l|}
\hline Meson & Data $[\mathrm{MeV}]$ & $f_{V}[\mathrm{MeV}]$ & $R=f_{V} / f_{V}^{\exp }$ & $\kappa[\mathrm{GeV}]$ \\
\hline$\rho^{0}$ & 154.7 & 130 & 0.84 & 0.6 \\
\hline$\omega$ & 45.8 & 43.3 & 0.95 & 0.6 \\
\hline$\phi$ & 76 & 63.2 & 0.83 & 0.6 \\
\hline$J / \psi$ & 277.6 & 201.1 & 0.72 & 1 \\
\hline$\Upsilon(1 s)$ & 238.4 & 142.2 & 0.60 & 1.37 \\
\hline
\end{tabular}

\section{Acknowledgments}

This work was supported by the DFG under Contract No. FA67/31-2 and No. GRK683, by Russian Science and Innovations Federal Agency under contract No 02.740.11.0238. This research is also part of the European Community-Research Infrastructure Integrating Activity "Study of Strongly Interacting Matter" (HadronPhysics2, Grant Agreement No. 227431) and of the President grant of Russia "Scientific Schools" No. 871.2008.2.

\section{References}

[1] T. Gutsche, V. E. Lyubovitskij and M. C. Tichy, Phys. Rev. D 79, 014036 (2009); Phys. Rev. D 80 , 014014 (2009).

[2] F. Giacosa, T. Gutsche, V. E. Lyubovitskij and A. Faessler, Phys. Rev. D 72, 114021 (2005); D 72, 094006 (2005); Phys. Lett. B 622, 277 (2005).

[3] S. J. Brodsky and G. F. de Teramond, Phys. Lett. B 582, 211 (2004); Phys. Rev. Lett. 94, 201601 (2005); 96, 201601 (2006); Phys. Rev. D 77, 056007 (2008).

[4] A. Vega and I. Schmidt, Phys. Rev. D 78, 017703 (2008); Phys. Rev. D 79, 055003 (2009).

[5] A. Vega, I. Schmidt, T. Branz, T. Gutsche and V. E. Lyubovitskij, Phys. Rev. D 80, 055014 (2009); T. Branz, T. Gutsche, V. E. Lyubovitskij, I. Schmidt and A. Vega, in preparation.

[6] G. Ecker, J. Gasser, H. Leutwyler, A. Pich and E. de Rafael, Phys. Lett. B 223, 425 (1989);

V. Cirigliano, G. Ecker, H. Neufeld and A. Pich, JHEP 0306, 012 (2003).

[7] J. M. Maldacena, Adv. Theor. Math. Phys. 2, 231 (1998) [Int. J. Theor. Phys. 38, 1113 (1999)]

[8] G. F. de Teramond, Invited talk at the Third Workshop of the APS Topical Group in Hadron Physics "GHP2009", http://www.fz-juelich.de/ikp/ghp2009/Program.shtml 